\title{
Neuroprotective mechanisms of 3-n-butylphthalide in neurodegenerative diseases (Review)
}

\author{
RIXIN LUO ${ }^{1}$, RUNQI WANGQIN ${ }^{2}$, LIHONG ZHU ${ }^{3}$ and WEI BI ${ }^{1,4}$ \\ ${ }^{1}$ Department of Neurology, The First Affiliated Hospital of Jinan University, Guangzhou, Guangdong 510632, P.R. China; \\ ${ }^{2}$ Department of Neurology, Duke University Medical Center, Durham, NC 27705, USA; \\ ${ }^{3}$ Department of Pathophysiology, School of Medicine, Jinan University; \\ ${ }^{4}$ Clinical Neuroscience Institute of Jinan University, \\ Guangzhou, Guangdong 510632, P.R. China
}

Received April 7, 2019; Accepted September 19, 2019

DOI: $10.3892 /$ br.2019.1246

\begin{abstract}
Since 3-n-butylphthalide (NBP) was approved by the China Food and Drug Administration for the treatment of acute ischemia stroke in 2002, a number of studies have investigated NBP worldwide. In recent years, NBP has also demonstrated potential as treatment of several neurodegenerative diseases, which has increased the interest in its mechanisms of protection and action. Clinical studies and studies that used cell or animal models, have directly demonstrated neuroprotective effects of NBP via the following mechanisms: i) Inhibiting the inflammatory reaction; ii) reducing mitochondrial oxidative stress; iii) regulating apoptosis and autophagy; iv) inducing resistance to endoplasmic reticulum stress; and v) decreasing abnormal protein deposition. Therefore, NBP may be a potential drug for neurodegenerative diseases, and it is particularly important to identify the mechanism of NBP as it may assist with the development of new drugs for neurodegeneration. The present review summarizes the neuroprotective mechanisms of NBP and discusses new perspectives and prospects. The aim of the current review is to provide a new summary regarding NBP and its associated mechanisms.
\end{abstract}

\section{Contents}

1. Introduction

2. NBP inhibits the inflammatory reaction

Correspondence to: Professor Lihong Zhu, Department of Pathophysiology, School of Medicine, Jinan University, 601 Huang $\mathrm{Pu}$ Street, Guangzhou, Guangdong 510632, P.R. China

E-mail: lhzhu@jnu.edu.cn

Dr Wei Bi, Department of Neurology, The First Affiliated Hospital of Jinan University, 613 Huang Pu Street, Guangzhou, Guangdong 510632, P.R. China

E-mail: biwei4762@sina.com

Key words: 3-n-butylphthalide, neuroprotective mechanism, neurodegenerative disease
3. NBP reduces mitochondrial oxidative stress

4. NBP regulates apoptosis and autophagy

5. NBP resists endoplasmic reticulum stress

6. NBP decreases abnormal protein deposition

7. Conclusion

\section{Introduction}

3-n-butylphthalide (NBP), approved by the China Food and Drug Administration for the treatment of acute ischemic stroke, is a type of compound isolated from the seeds of Chinese celery (1). The molecular structure of NBP is presented in Fig. 1. Therapy using NBP has been recommended by Chinese guidelines for acute ischemic stroke (2). A randomized double-blind trial (clinical trial no. ChiCTR-TRC-09000483) reported that NBP significantly improves clinical outcomes, including the modified Rankin Scale (3) and National institute of Health Stroke Scale scores (4), of patients who experienced ischemic stroke (5). In addition, a study demonstrated that NBP therapy persistently increases the level of endothelial progenitor cells in peripheral blood, ameliorate cerebral blood flow and improve neuronal functions (6). Furthermore, NBP has been reported to be a safe treatment for cerebral ischemia stroke (5-7). A study has indicated that NBP exhibits protective effects in several neurodegenerative diseases (8). However, to the best of our knowledge, the neuroprotective mechanism of NBP remains unclear. Therefore, the present review discusses the potential mechanism of neuroprotective effects of NBP. The aim of the current review is to provide further understanding regarding the advances of NBP.

\section{NBP inhibits the inflammatory reaction}

Inflammation, a complex biological response to injury, is associated with neurodegenerative diseases, including Alzheimer's disease, Parkinson's disease (PD), multiple sclerosis, amyotrophic lateral sclerosis, traumatic brain injury (TBI) and more (9-11). NBP has exhibited anti-inflammatory effects in various models of these diseases and certain mechanisms have been identified. NBP has been reported to reduce 
the inflammatory reaction by inhibiting nucleotide binding oligomerization domain like receptor protein 3-inflammasome microglia activation and mitigating the Alzheimer's-like pathology via the nuclear factor erythroid-2-related factor 2-thioredoxin-interacting protein-TXNIP-thioredoxin axis in an APP/PS1 mouse model $(12,13)$. Furthermore, NBP inhibited the inflammatory reaction in lipopolysaccharide (LPS)-induced rats via inhibition of c-Jun N-terminal kinase activation and the NF- $\mathrm{kB}$ pathway $(14,15)$. NBP was reported to improve dyskinesia in a LPS-induced PD mouse model via a reduction in the loss of dopaminergic neurons, activation of mouse microglia, an increase in TNF- $\alpha$ levels and $\alpha$-synuclein deposition in the black substantia of the mouse midbrain (16). Additionally, NBP-treatment reduces NF- $\kappa \mathrm{B}$ activation following TBI (17), and NBP also inhibits the inflammatory reaction via the same pathway in spontaneously hypertensive rats (18). Notably, a number of studies have indicated that NBP inhibits the inflammatory reaction in other neuroassociated experimental models, such as an experimental model of autoimmune encephalomyelitis of microglia or autoimmune myositis in guinea pigs $(19,20)$. In addition, NBP-treatment has been demonstrated to significantly ameliorate cerebral ischemia reperfusion-induced brain injury of Sprague-Dawley (SD) rats by inhibiting toll like receptor $4 / \mathrm{NF}-\mathrm{\kappa B}$-associated inflammation (21). NBP attenuates advanced glycation end products-induced endothelial dysfunction by ameliorating inflammatory responses (22). In summary, there is some understanding regarding the mechanism of NBP in the inhibition of inflammation.

\section{NBP reduces mitochondrial oxidative stress}

Mitochondria, the site of oxidative metabolism in eukaryotes, produce energy through the oxidation of carbohydrates, fats and amino acids (23). Therefore, mitochondrial dysfunction in the form of oxidative stress may contribute to the pathogenesis of various neurodegenerative diseases (24). Oxidative stress is considered a condition that is caused by an imbalance between pro- and antioxidant factors, which leads to molecular and cellular damage (25). Oxidative stress serves an essential role in the development of age-related diseases (26). NBP exhibits a cumulative beneficial effect on the process of mitochondrial damage (27). This section will discuss the mechanisms involved in mitochondrial oxidative stress.

Recently, NBP exhibited a powerful effect on antioxidant stress in some different models. NBP inhibited oxidative stress in K141N-induced SH-SY5Y cells and in LPS-induced rats through activation of the Kelch-like ECH-associating protein 1 Nrf2-related factor 2-antioxidant response element signaling pathway $(15,28)$. Similarly, NBP reduced oxidative damage to provide neuroprotection in mice following TBI and in rats following carbon monoxide poisoning $(29,30)$. In addition, NBP protects against cerebral ischemia-reperfusion injury by decreasing antioxidant stress via the ERK signaling pathway (31). NBP also protects against $\mathrm{H}_{2} \mathrm{O}_{2}$-induced injury in neural stem cells by activation of the PI3K/Akt and the Mash1 signaling pathways (32). Furthermore, NBP has been reported to increase superoxide dismutase and catalase activity, and reduce malondialdehyde activity in the experimental autoimmune myositis (EAM) model, NBP directly protects muscle

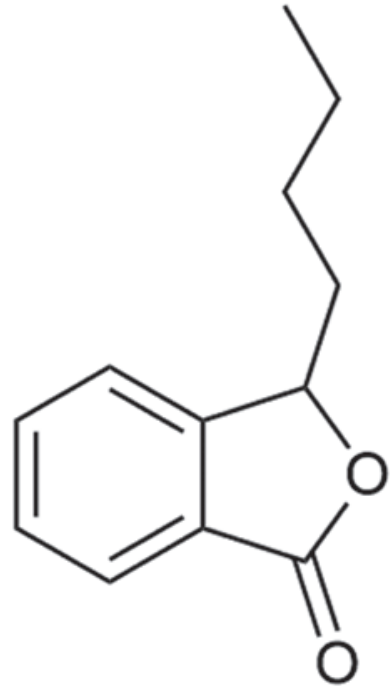

Figure 1. Molecular structure of 3-n-butylphthalide.

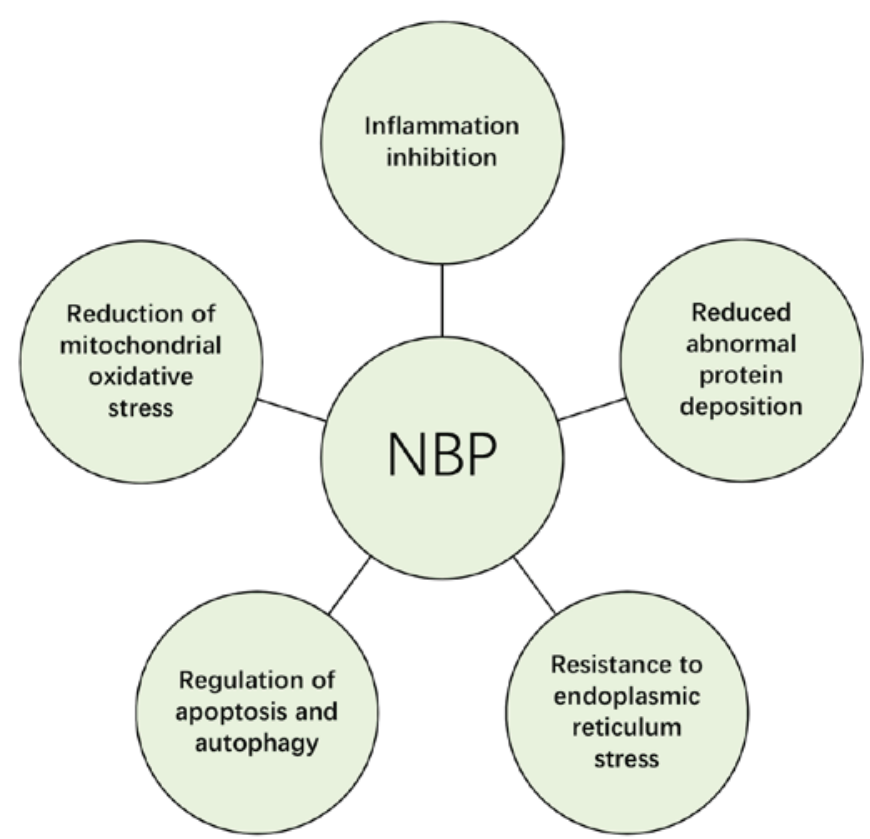

Figure 2. Neuroprotective mechanisms of NBP. NBP, 3-n-butylphthalide.

mitochondria and muscle cells from oxidative damage (33). However, the protective effect of NBP on mitochondrial function is not only limited to neurodegeneration, but also appears in cardiovascular diseases. A study suggested that NBP exerts a cardioprotective effect on cardiac ischemic injury via the regulation of mitochondrial function both using in vivo and in vitro experiments (34). In summary, the antioxidant effect of NBP has been widely recognized.

\section{NBP regulates apoptosis and autophagy}

Apoptosis and autophagy are basic biological phenomena of cells, which serve essential roles in removing abnormal cells in multicellular organisms. Disorders in the apoptosis and autophagy processes may cause the occurrence of neuropathy (35). The neuroprotective effect of NBP via the regulation 
Table I. Neuroprotective mechanisms of 3-n-butylphthalide.

A, Inflammation inhibition

\begin{tabular}{|c|c|c|c|c|}
\hline Author, year & Study subject & Method & Molecular mechanism & Refs. \\
\hline Wang et al, 2018 & $\begin{array}{l}\text { APP/PS1 mice } \\
\text { A172, SH-SY5Y }\end{array}$ & $\begin{array}{l}\text { Transgenic } \\
\text { LPS induced }\end{array}$ & $\begin{array}{l}\text { NLRP3 inflammasome activation inhibition } \\
\text { NLRP3 inflammasome activation inhibition }\end{array}$ & (13) \\
\hline Yang et al, 2018 & $\mathrm{SD}$ rats & LPS induced & $\mathrm{NF}-\kappa \mathrm{B}$ pathway inhibition & (14) \\
\hline Zhao et al, 2016 & C57BL/6 mice & LPS induced & Downregulation of JNK activation & $(15)$ \\
\hline Zhao et al, 2017 & C57BL/6 mice & Traumatic brain injury & $\mathrm{NF}-\kappa \mathrm{B}$ pathway inhibition & $(17)$ \\
\hline Wang et al, 2018 & EAE & $\begin{array}{l}\text { Neuroantigen-specific } \\
\text { proinflammatory } \mathrm{T} \text { cells induced }\end{array}$ & Suppression of PGAM5 & (19) \\
\hline Zhang et al, 2016 & SD rats & $\begin{array}{l}\text { Cerebral ischemia reperfusion } \\
\text { induced }\end{array}$ & Increased HGF expression & $(21)$ \\
\hline Liu et al, 2017 & HUVECs & $\begin{array}{l}\text { Advanced glycation end } \\
\text { product induced }\end{array}$ & RAGE/NF- $\kappa$ B pathway inhibition & $(22)$ \\
\hline
\end{tabular}

$\mathrm{B}$, Reduction of mitochondrial oxidative stress

\begin{tabular}{|c|c|c|c|c|}
\hline Author, year & Study subject & Method & Molecular mechanism & Refs. \\
\hline Yang et al, 2017 & SH-SY5Y & Missense mutations & Increased Nrf2 expression & $(28)$ \\
\hline Liu et al, 2017 & ICR mice & Traumatic brain injury & Nrf2-ARE pathway activation & $(29)$ \\
\hline Li et al, 2015 & $\mathrm{SD}$ rats & Carbon monoxide poisoned & Keap1/Nrf2 pathway activation & $(30)$ \\
\hline Zhu et al, 2018 & ICR mice & $\begin{array}{l}\text { Cerebral ischemia reperfusion } \\
\text { injury }\end{array}$ & ERK signaling inhibition & $(31)$ \\
\hline Wang et al, 2018 & NSCs from SD rats & Hydrogen peroxide induced & PI3K/Akt and Mash1 pathway activation & $(32)$ \\
\hline Chen et al, 2017 & Guinea pigs & $\begin{array}{l}\text { Experimental autoimmune } \\
\text { myositis }\end{array}$ & $\begin{array}{l}\text { Enhanced } \mathrm{Na}^{+}-\mathrm{K}^{+} \text {and } \\
\mathrm{Ca} 2^{+}-\mathrm{Mg}^{2+} \text { ATPase activities }\end{array}$ & $(33)$ \\
\hline Tian et al, 2017 & $\mathrm{H} 9 \mathrm{C} 2$ & Hydrogen peroxide induced & Enhanced Nrf-1 and TFAM expression & $(34)$ \\
\hline
\end{tabular}

C, Regulation of apoptosis and autophagy

\begin{tabular}{|c|c|c|c|c|}
\hline Author, year & Study subject & Method & Molecular mechanism & Refs. \\
\hline Zhao et al, 2017 & C57BL/6 mice & Traumatic brain injury & Downregulated caspase- 3 and -9 expression & $(17)$ \\
\hline Liu et al, 2017 & HUVECs & $\begin{array}{l}\text { Advanced glycation end } \\
\text { product induced }\end{array}$ & Regulation of Bcl-2 expression & (22) \\
\hline Lei et al, 2014 & SH-SY5Y & $\beta$-amyloid induced & $\begin{array}{l}\text { Regulation of Bcl-2, caspase- } 3 \text { and } \\
-9 \text { expression }\end{array}$ & $(37)$ \\
\hline $\mathrm{Xu}$ et al, 2017 & C57BL/6 mice & $\begin{array}{l}\text { Repeated cerebral ischemia } \\
\text { reperfusion }\end{array}$ & Bcl-2/Bax elevation & $(38)$ \\
\hline Xiang et al, 2014 & APP/PS1 mice & Transgenic & BDNF/TrkB/PI3K/Akt pathway regulation & $(39)$ \\
\hline
\end{tabular}

$\mathrm{D}$, Resistance to endoplasmic reticulum stress

\begin{tabular}{|c|c|c|c|}
\hline Author, year & Study subject & Method & Molecular mechanism \\
\hline Liao et al, 2018 & SD rats & Doxorubicin induced & $\begin{array}{l}\text { GRP } 78, \text { CHOP and caspase- } 12 \text { expression } \\
\text { regulation }\end{array}$ \\
\hline Niu et al, 2018 & $\mathrm{SD}$ rats & $\begin{array}{l}\text { Bilateral surgical ligation of } \\
\text { common carotid arteries }\end{array}$ & $\begin{array}{l}\text { GRP } 78, \text { CHOP and caspase- } 12 \\
\text { expression regulation }\end{array}$ \\
\hline \multirow[t]{2}{*}{ Zheng et al, 2017} & $\mathrm{SD}$ rats & Laminectomy performed at $\mathrm{T} 9$ & $\begin{array}{l}\text { ATF-4, ATF-6, XBP-1, PDI, GRP78, } \\
\text { CHOP and cleaved-caspase } 12 \text { attenuation }\end{array}$ \\
\hline & HBMECs & Thapsigargin induced & $\begin{array}{l}\text { ATF-4, ATF-6, XBP-1, PDI, GRP78, } \\
\text { CHOP and cleaved-caspase } 12 \text { attenuation }\end{array}$ \\
\hline
\end{tabular}

Refs. 
Table I. Continued.

$\mathrm{D}$, Resistance to endoplasmic reticulum stress

\begin{tabular}{llll}
\hline Author, year & Study subject & Method & Molecular mechanism \\
\hline He et al, 2017 & SD rats & Laminectomy performed at T9 & ATF-4, ATF-6, XBP-1, PDI, GRP78, \\
& PC12 & Thapsigargin induced & ATF-4, ATF-6, XBP-1, PDI, GRP78, \\
& & CHOP and cleaved-caspase 12 attenuation \\
\hline
\end{tabular}

E, Reduced abnormal protein deposition

\begin{tabular}{|c|c|c|c|c|}
\hline Author, year & Study subject & Method & Molecular mechanism & Refs. \\
\hline Peng et al, 2010 & $3 \times T g-A D$ mice & Transgenic & $\begin{array}{l}\text { Direction of APP processing towards a } \\
\text { non-amyloidogenic pathway }\end{array}$ & (47) \\
\hline Peng et al, 2012 & $\mathrm{~A} \beta \mathrm{PP} / \mathrm{PS} 1$ mice & Transgenic & Tau hyperphosphorylation inhibition & $(48)$ \\
\hline Chen et al, 2018 & C57BL/6 mice & LPS induced & Reduction of $\alpha$-synuclein deposition & (16) \\
\hline Huang et al, 2010 & PC12 & $\mathrm{MPP}^{+}$toxicity induced & Reduction of $\alpha$-synuclein deposition & $(49)$ \\
\hline
\end{tabular}

of apoptosis and autophagy has been demonstrated. Treatment with NBP has been reported to reduce apoptotic cell death by increasing the levels of cleaved caspase- 3 and caspase- 9 following TBI (17). Furthermore, NBP blocks neural apoptosis in areas surrounding cortical contusions on the brain that are induced by TBI (29). The neuroprotective mechanism of NBP involves the mitochondrial apoptotic pathway. NBP inhibits HSPB8 K141N mutation-induced neurotoxicity, attenuates $\beta$-amyloid-induced toxicity in SH-SY5Y cells, and protects rat cardiomyocytes from ischemia or reperfusion through regulating mitochondrion-mediated apoptosis $(28,36,37)$. Furthermore, certain studies have demonstrated the inhibition of apoptosis by NBP via the Akt pathway. One study reported that NBP activates Akt/mTOR signaling to inhibit neuronal apoptosis and autophagy in mice with repeated cerebral ischemia reperfusion injury (38). Another study demonstrated that NBP improves cognitive impairment of APP/PS1 mice by inhibiting apoptosis via the PI3K/AKT pathway (39). Additionally, NBP reduces the number of apoptotic cells by regulating Bcl-2 in HUVECs and an EAM model $(22,33)$.

\section{NBP resists endoplasmic reticulum stress}

ERS is characterized by incorrect folding and aggregation of unfolded proteins in the endoplasmic reticulum lumen and a disturbance of the calcium balance, which can activate the unfolded protein response and lead to disturbance of the cell function and cell death (40). In recent years, certain studies have reported an anti-ERS effect of NBP. One study demonstrated that NBP inhibits doxorubicin-induced ERS in SD rats (41).
In addition, NBP alleviates vascular cognitive impairment by regulating ERS and the Sonic hedgehog/Patched homolog 1 signaling pathway in SD rats (42). Both of these studies agreed that NBP attenuates ERS through regulating the expression of 78-kDa glucose-regulated protein (GRP78), CCAAT-enhancer binding protein homologous protein (CHOP) and caspase-12. Furthermore, NBP also inhibits ERS by attenuating activating transcription factory (ATF)-4, ATF-6, X-box binding protein 1, protein disulfide isomerase, GRP78, CHOP and cleaved-caspase-12 in a spinal cord injury (SCI) model, which may improve functional recovery and prevent disruption of the blood-spinal cord barrier $(43,44)$. However, this mechanism has only recently been identified; therefore, there is limited literature about it. Further research on this mechanism may lead to new findings.

\section{NBP decreases abnormal protein deposition}

Abnormal protein deposition is closely associated with numerous neurodegenerative diseases (45), such as Alzheimer's disease, which is associated with amyloid- $\beta$ $(\mathrm{A} \beta)$ and tau proteins; and PD, which is associated with $\alpha$-synuclein (46). A study has demonstrated that NBP significantly reduces total cerebral $A \beta$ plaque deposition and lowers $\mathrm{A} \beta$ levels in brain homogenates in a triple-transgenic mouse model of Alzheimer's disease via directing amyloid precursor protein processing toward a non-amyloidogenic pathway (47). Furthermore, NBP treatment inhibited tau hyperphosphorylation in A $\beta P P / P S 1$ mice, which may improve cognitive impairment (48). NBP enhances a 1-methyl-4-phenylpyridini- 
umion-induced cellular model and a LPS-induced mice model of PD via reducing the accumulation of $\alpha$-synuclein $(16,49)$. However, the molecular mechanisms of how NBP reduces the accumulation of $\alpha$-synuclein and inhibits tau hyperphosphorylation remain unclear. Furthermore, to the best of our knowledge, there is no associated study that provides the clinical evidence that NBP is effective in multiple sclerosis or Lewy body dementia via attenuating abnormal protein deposition. Potentially, new findings can be revealed in additional neurodegenerative diseases.

\section{Conclusion}

In summary, current studies suggest that NBP serves a neuroprotective role through inhibiting inflammation, protecting mitochondrial function, alleviating oxidative stress, regulating apoptosis, resisting ERS and decreasing the abnormal protein deposition (Fig. 2). Details on specific molecular mechanisms are presented in Table I. Taken together, it is suggested that NBP provides a promising therapeutic strategy for neurodegenerative diseases. In further studies, the mechanism of action of NBP may be further clarified, and the understanding regarding its potential uses may be expanded.

\section{Acknowledgements}

Not applicable.

\section{Funding}

This study was supported by grants from the Natural Science Foundation of China (grant no. 81371442), the Training program for outstanding young teachers in higher education institutions of Guangdong Province (grant no. YQ2015024) and the Fundamental Research Funds for the Central Universities (grant no. 21617482).

\section{Availability of data and materials}

All data generated or analyzed during this study are included in this published article.

\section{Authors' contributions}

$\mathrm{RL}$ was a major contributor in writing the manuscript. RL, RW, LZ and WB contributed to researching data, discussing content and editing the manuscript. All authors read and approved the final version of the manuscript.

\section{Ethics approval and consent to participate}

Not applicable.

\section{Patient consent for publication}

Not applicable.

\section{Competing interests}

The authors declare that they have no competing interests.

\section{References}

1. Xu ZQ, Zhou Y, Shao BZ, Zhang JJ and Liu C: A Systematic Review of Neuroprotective Efficacy and Safety of DL-3-N-Butylphthalide in Ischemic Stroke. Am J Chin Med 47: 507-525, 2019.

2. Chinese Society of Cerebral Blood Flow and Metabolism: The Chinese guidelines for the evaluation and management of cerebral collateral circulation in ischemic stroke (2017). Zhonghua Nei Ke Za Zhi 56: 460-471, 2017 (In Chinese).

3. Banks JL and Marotta CA: Outcomes validity and reliability of the modified Rankin scale: implications for stroke clinical trials: a literature review and synthesis. Stroke 38: 1091-1096, 2007.

4. Heldner MR, Zubler C, Mattle HP, Schroth G, Weck A, Mono ML, Gralla J, Jung S, El-Koussy M, Lüdi R, et al: National Institutes of Health stroke scale score and vessel occlusion in 2152 patients with acute ischemic stroke. Stroke 44: 1153-1157, 2013.

5. Cui LY, Zhu YC, Gao S, Wang JM, Peng B, Ni J, Zhou LX, He J and Ma XQ: Ninety-day administration of dl-3-n-butylphthalide for acute ischemic stroke: A randomized, double-blind trial. Chin Med J (Engl) 126: 3405-3410, 2013.

6. Zhao H, Yun W, Zhang Q, Cai X, Li X, Hui G, Zhou X and Ni J: Mobilization of Circulating Endothelial Progenitor Cells by dl-3-n-Butylphthalide in Acute Ischemic Stroke Patients. J Stroke Cerebrovasc Dis 25: 752-760, 2016.

7. Zhang C, Zhao S, Zang Y, Gu F, Mao S, Feng S, Hu L and Zhang C: The efficacy and safety of Dl-3n-butylphthalide on progressive cerebral infarction: A randomized controlled STROBE study. Medicine (Baltimore) 96: e7257, 2017.

8. Huang L, Wang S, Ma F, Zhang Y, Peng Y, Xing C, Feng Y, Wang $X$ and Peng Y: From stroke to neurodegenerative diseases: The multi-target neuroprotective effects of 3-n-butylphthalide and its derivatives. Pharmacol Res 135: 201-211, 2018.

9. Skaper SD, Facci L, Zusso M and Giusti P: An Inflammation-Centric View of Neurological Disease: Beyond the Neuron. Front Cell Neurosci 12: 72, 2018.

10. Liu J and Wang F: Role of Neuroinflammation in Amyotrophic Lateral Sclerosis: Cellular Mechanisms and Therapeutic Implications. Front Immunol 8: 1005, 2017.

11. Niu F, Sharma A,Feng L, Ozkizilcik A, Muresanu DF, Lafuente JV, Tian ZR, Nozari A and Sharma HS: Nanowired delivery of DL-3-n-butylphthalide induces superior neuroprotection in concussive head injury. Prog Brain Res 245: 89-118, 2019.

12. Zhang Y, Huang LJ, Shi S, Xu SF, Wang XL and Peng Y: L-3-n-butylphthalide Rescues Hippocampal Synaptic Failure and Attenuates Neuropathology in Aged APP/PS1 Mouse Model of Alzheimer's Disease. CNS Neurosci Ther 22: 979-987, 2016.

13. Wang $\mathrm{CY}, \mathrm{Xu}$ Y, Wang $\mathrm{X}$, Guo $\mathrm{C}$, Wang $\mathrm{T}$ and Wang ZY: D1-3-n-Butylphthalide Inhibits NLRP3 Inflammasome and Mitigates Alzheimer's-Like Pathology via Nrf2-TXNIP-TrX Axis. Antioxid Redox Signal 30: 1411-1431, 2018.

14. Yang M, Dang R, Xu P, Guo Y, Han W, Liao D and Jiang P: Dl-3-n-Butylphthalide improves lipopolysaccharide-induced depressive-like behavior in rats: Involvement of Nrf2 and NF- $\mathrm{BB}$ pathways. Psychopharmacology (Berl) 235: 2573-2585, 2018.

15. Zhao CY, Lei H, Zhang Y, Li L, Xu SF, Cai J, Li PP, Wang L, Wang XL and Peng Y: L-3-n-Butylphthalide attenuates neuroinflammatory responses by downregulating JNK activation and upregulating Heme oxygenase-1 in lipopolysaccharide-treated mice. J Asian Nat Prod Res 18: 289-302, 2016.

16. Chen Y, Jiang M, Li L, Ye M, Yu M, Zhang L, Ge B, Xu W and Wei D: DL-3-n-butylphthalide reduces microglial activation in lipopolysaccharide-induced Parkinson's disease model mice. Mol Med Rep 17: 3884-3890, 2018.

17. Zhao Y, Lee JH, Chen D, Gu X, Caslin A, Li J, Yu SP and Wei L: DL-3-n-butylphthalide induced neuroprotection, regenerative repair, functional recovery and psychological benefits following traumatic brain injury in mice. Neurochem Int 111: 82-92, 2017.

18. Zhu J,Zhang Y and Yang C: Protective effect of 3-n-butylphthalide against hypertensive nephropathy in spontaneously hypertensive rats. Mol Med Rep 11: 1448-1454, 2015.

19. Wang Y, Bi Y, Xia Z, Shi W, Li B, Li B, Chen L and Guo L: Butylphthalide ameliorates experimental autoimmune encephalomyelitis by suppressing PGAM5-induced necroptosis and inflammation in microglia. Biochem Biophys Res Commun 497: 80-86, 2018.

20. Chen J, Wang J, Zhang J and Pu C: Effect of butylphthalide intervention on experimental autoimmune myositis in guinea pigs. Exp Ther Med 15: 152-158, 2018. 
21. Zhang P, Guo ZF, Xu YM, Li YS and Song JG: N-Butylphthalide (NBP) ameliorated cerebral ischemia reperfusion-induced brain injury via HGF-regulated TLR4/NF- $\kappa \mathrm{B}$ signaling pathway. Biomed Pharmacother 83: 658-666, 2016.

22. Liu CY, Zhao ZH, Chen ZT, Che CH, Zou ZY, Wu XM, Chen SG, Li YX, Lin HB, Wei XF, et al: DL-3-n-butylphthalide protects endothelial cells against advanced glycation end product-induced injury by attenuating oxidative stress and inflammation responses. Exp Ther Med 14: 2241-2248, 2017.

23. Schapira AHV: Mitochondrial diseases. Lancet 379: 1825-1834, 2012.

24. Arun S, Liu L and Donmez G: Mitochondrial Biology and Neurological Diseases. Curr Neuropharmacol 14: 143-154, 2016.

25. Hybertson BM, Gao B, Bose SK and McCord JM: Oxidative stress in health and disease: The therapeutic potential of Nrf2 activation. Mol Aspects Med 32: 234-246, 2011.

26. Tan BL, Norhaizan ME, Liew WP and Sulaiman Rahman H: Antioxidant and Oxidative Stress: A Mutual Interplay in Age-Related Diseases. Front Pharmacol 9: 1162, 2018.

27. Abdoulaye IA and Guo YJ: A Review of Recent Advances in Neuroprotective Potential of 3-N-Butylphthalide and Its Derivatives. BioMed Res Int 2016: 5012341, 2016.

28. Yang XD, Cen ZD, Cheng HP, Shi K, Bai J, Xie F, Wu HW, Li BB and Luo W: L-3-n-Butylphthalide Protects HSPB8 K141N Mutation-Induced Oxidative Stress by Modulating the Mitochondrial Apoptotic and Nrf2 Pathways. Front Neurosci 11: 402, 2017.

29. Liu Z, Wang H, Shi X, Li L, Zhou M, Ding H, Yang Y, Li X and Ding K: DL-3-n-Butylphthalide (NBP) Provides Neuroprotection in the Mice Models After Traumatic Brain Injury via Nrf2-ARE Signaling Pathway. Neurochem Res 42: 1375-1386, 2017.

30. Li Q, Cheng Y, Bi M, Lin H, Chen Y, Zou Y, Liu Y, Kang H and Guo Y: Effects of N-butylphthalide on the activation of Keap1/Nrf-2 signal pathway in rats after carbon monoxide poisoning. Environ Toxicol Pharmacol 40: 22-29, 2015.

31. Zhu BL, Xie CL, Hu NN, Zhu XB and Liu CF: Inhibiting of GRASP65 Phosphorylation by DL-3-N-Butylphthalide Protects against Cerebral Ischemia-Reperfusion Injury via ERK Signaling. Behav Neurol 2018: 5701719, 2018.

32. Wang S, Huang L, Zhang Y,Peng Y, Wang X and Peng Y: Protective Effects of L-3-n-Butylphthalide Against H2O2-Induced Injury in Neural Stem Cells by Activation of PI3K/Akt and Mash1 Pathway. Neuroscience 393: 164-174, 2018.

33. Chen J, Wang J, Zhang J and Pu C: 3-n-Butylphthalide reduces the oxidative damage of muscles in an experimental autoimmune myositis animal model. Exp Ther Med 14: 2085-2093, 2017.

34. Tian X, He W, Yang R and Liu Y: Dl-3-n-butylphthalide protects the heart against ischemic injury and $\mathrm{H} 9 \mathrm{c} 2$ cardiomyoblasts against oxidative stress: Involvement of mitochondrial function and biogenesis. J Biomed Sci 24: 38, 2017.

35. Booth LA, Tavallai S, Hamed HA, Cruickshanks N and Dent P The role of cell signalling in the crosstalk between autophagy and apoptosis. Cell Signal 26: 549-555, 2014.

36. Wang YG, Li Y, Wang CY, Ai JW, Dong XY, Huang HY, Feng ZY, Pan YM, Lin Y, Wang BX, et al: L-3-n-Butylphthalide protects rats' cardiomyocytes from ischaemia/reperfusion-induced apoptosis by affecting the mitochondrial apoptosis pathway. Acta Physiol (Oxf) 210: 524-533, 2014.

37. Lei H, Zhao CY, Liu DM, Zhang Y, Li L, Wang XL and Peng Y: $1-3$-n-Butylphthalide attenuates $\beta$-amyloid-induced toxicity in neuroblastoma SH-SY5Y cells through regulating mitochondrion-mediated apoptosis and MAPK signaling. J Asian Nat Prod Res 16: 854-864, 2014.
38. Xu J, Huai Y, Meng N, Dong Y, Liu Z, Qi Q, Hu M, Fan M, Jin W and Lv P: L-3-n-Butylphthalide Activates Akt/mTOR Signaling, Inhibits Neuronal Apoptosis and Autophagy and Improves Cognitive Impairment in Mice with Repeated Cerebral Ischemia-Reperfusion Injury. Neurochem Res 42: 2968-2981, 2017.

39. Xiang J, Pan J, Chen F, Zheng L, Chen Y, Zhang S and Feng W: L-3-n-butylphthalide improves cognitive impairment of APP/PS1 mice by BDNF/TrkB/PI3K/AKT pathway. Int J Clin Exp Med 7: 1706-1713, 2014.

40. Iurlaro R and Muñoz-Pinedo C: Cell death induced by endoplasmic reticulum stress. FEBS J 283: 2640-2652, 2016.

41. Liao D, Xiang D, Dang R, Xu P, Wang J, Han W, Fu Y, Yao D, Cao L and Jiang P: Neuroprotective Effects of dl-3-n-Butylphthalide against Doxorubicin-Induced Neuroinflammation, Oxidative Stress, Endoplasmic Reticulum Stress, and Behavioral Changes. Oxid Med Cell Longev 2018: 9125601, 2018.

42. Niu XL, Jiang X, Xu GD, Zheng GM, Tang ZP, Yin N, Li XQ, Yang YY and Lv PY: DL-3-n-butylphthalide alleviates vascular cognitive impairment by regulating endoplasmic reticulum stress and the Shh/Ptch1 signaling-pathway in rats. J Cell Physiol 234: 12604-12614, 2018.

43. Zheng B, Zhou Y, Zhang H, Yang G, Hong Z, Han D, Wang Q, He Z, Liu Y, Wu F, et al: Dl-3-n-butylphthalide prevents the disruption of blood-spinal cord barrier via inhibiting endoplasmic reticulum stress following spinal cord injury. Int J Biol Sci 13: 1520-1531, 2017.

44. He Z, Zhou Y, Huang Y, Wang Q, Zheng B, Zhang H, Li J, Liu Y, Wu F, Zhang X, et al: Dl-3-n-butylphthalide improves functional recovery in rats with spinal cord injury by inhibiting endoplasmic reticulum stress-induced apoptosis. Am J Trans Res 9: 1075-1087, 2017.

45. Nonaka T, Masuda-Suzukake M and Hasegawa M: Molecular mechanisms of the co-deposition of multiple pathological proteins in neurodegenerative diseases. Neuropathology 38: 64-71, 2018

46. Goedert M: NEURODEGENERATION. Alzheimer's and Parkinson's diseases: The prion concept in relation to assembled $\mathrm{A} \beta$, tau, and $\alpha$-synuclein. Science 349: 1255555, 2015.

47. Peng Y, Sun J, Hon S, Nylander AN, Xia W, Feng Y, Wang X and Lemere CA: L-3-n-butylphthalide improves cognitive impairment and reduces amyloid-beta in a transgenic model of Alzheimer's disease. J Neurosci 30: 8180-8189, 2010.

48. Peng Y, Hu Y, Xu S, Li P, Li J, Lu L, Yang H, Feng N, Wang L and Wang X: L-3-n-butylphthalide reduces tau phosphorylation and improves cognitive deficits in A $\beta \mathrm{PP} / \mathrm{PS} 1-\mathrm{Alzh}$ imer's transgenic mice. J Alzheimers Dis 29: 379-391, 2012.

49. Huang JZ, Chen YZ, Su M, Zheng HF, Yang YP, Chen J and Liu CF: dl-3-n-Butylphthalide prevents oxidative damage and reduces mitochondrial dysfunction in an $\mathrm{MPP}(+)$-induced cellular model of Parkinson's disease. Neurosci Lett 475: 89-94, 2010 .

This work is licensed under a Creative Commons Attribution-NonCommercial-NoDerivatives 4.0 International (CC BY-NC-ND 4.0) License. 